\title{
An Analysis of Queueing Model with a Multiple System Governed by a Quasi-Birth Death Process and PH Service and PH Repair
}

\author{
M. Reni Sagaya $\operatorname{Raj}^{1}$, B. Chandrasekar ${ }^{2}$ \\ ${ }^{1,2}$ Department of Mathematics, Sacred Heart College(Autonomous), Tirupattur - 635601, Vellore District, Tamil Nadu, S.India
}

\begin{abstract}
In this paper we study the multiple systems of repair problem and present $N$-system with one working and the other systems are in repair, in standby or waiting repair. When the working system can fails it goes to repair and instantaneously a standby system becomes the working one. Standby system stands to system when the original system is on under repair. The systems are repair following the arrival order. We let the time between service and repair times have phase-type distribution. The working system has a life time governed by a phase-type distribution. We show the process that governs the system is a quasi-birth and death process, we perform steady state analysis of this model. A numerical example is presented and the expressions calculated through the paper are computationally implemented using the Matlab programme.
\end{abstract}

Keywords: Repairman problem, Phase-type distribution, Quasi-Birth and death process, Matrix Geometric Method, Conditional probability of failure, Stationary distribution.

\section{Introduction}

We consider an $\mathrm{N}$-system and repairable standby system with single repair facility. There is one working server and the others are in repair, in standby or waiting for repairing. Only the working system can fail. Upon failure, it goes to repair and is immediately replaced by a standby server that become the working system. We let time between service and repair times follows phase-type distribution. If at failure of a system all repair person are busy then the failed system has to wait until a repair person is available. Repair is as good as new. The queue discipline is first come first served. The life time of the working system has a distribution function $F(t)$. The distribution function of the repair time is given by $G(t)$. Both of these random times are independent. We consider a multiple system involving PH-type distribution and under the assumptions that we established for the system. It is limited the literature on reliability for this distribution class.

Neuts.M.F and Meiee.K.S [8] studied a two unit system with operational and repair times follows phase-type distribution in cold standby and with two repair facilities. Chakravarthy.S [1] studied a similar problem from stand point of queue theory, considering the queue with single repair facility service when there are $n$ machines in parallel with operational times exponentially distributed and when the repair time follows a phase-type distribution. Chakravarthy.S and Natarajan.R [2] studied an n-system in a random environment modelled by a Markov process with generator by blocks, similar to the ones we applied when consider phase-type distribution. We show that the above mentioned multiply $\mathrm{N}$-system in which phase-type distribution are involved is governed by a quasi-birth and death process(QBD). These process extend the classical birth and death processes to the vectorial case, and the tri-diagonal generator of parameters is substituted by block tri-diagonal matrix. Several authors have considered QBD processes from different view point. Neuts [7] introduced one such process into queueing theory, showing that the stationary probability vector of a QBD process with infinite states can be expressed in terms of a matrix that plays an important role in the theory(rate matrix). Neuts.M.F, Perez-Ocon.R and Terres Castro.I [9] studied repairable models with operating and repairs times governed by phase-type distribution.

Latouche.G and Ramaswami.V [4] introduced a logarithmic reduction algorithm for calculating the rate matrix. Phasetype distribution are recognized as very good approximations for most general distribution encountered in the real life. Ramaswami.V and Taylor.A.G [10] studied the rate of operators in level dependent quasi-birth and death processes with a countable number of phases. Thus works, and references therein, complete the analysis of QBD processes at present. We have applied some of the general formulae from $[11,13,14]$ to our system. We consider a multiple system involving PH-type distribution and under the assumption that we established for the system. For this system, the stationary probability vector, the availability and the rate of occurrence of failures are calculate. A numerical examples are presented and the expressions calculated through the paper are computationally implemented using the matlab programme.

This paper is arranged as follows: In section 2, we analysis the PH-distribution. In Section 3, we gives the model description and equation covering of the system. In section 4, present a quasi-birth and death(QBD) model formulation. In section 5 , we obtain the stationary probability vector of the system size. In section 6 , present queue length and waiting time distribution of repair for the system. In section 7, and 8, we give the availability of the system and rate of occurrence of failure of system. In section 9, we give the numerical examples. Conclusion are given in section 10 .

We summarize the following definitions used in the paper. 


\section{International Journal of Science and Research (IJSR) \\ ISSN (Online): 2319-7064}

Index Copernicus Value (2013): 6.14 | Impact Factor (2014): 5.611

\section{Phase-Type distribution}

Definition Continuous Phase-type distribution:

1 Let $\left\{X_{n}: n \in N\right\}$ be a Markov chain as defined with state space $S=\{1,2,3, \ldots, m, m+1\}$, where the first $m$ states are transient and the last state is absorbing, and transition probability matrix

$$
\Theta=\left\lfloor\begin{array}{ll}
T & t^{\prime} \\
0 & 0
\end{array}\right\rfloor
$$

where $T$ is a square matrix of dimension $m, t^{\prime}$ is a column vector and is a row vector of dimension $m$. Since $Q$ is a generator matrix, we have $T_{i j} \geq 0$ and $t_{i}^{\prime} \geq 0 \forall i, j \in S$ and $T 1+t^{\prime}=1$. Where 1 is the column vector of ones of the appropriate dimension $m$. The probability distribution of the initial state is denoted with the row vector $\left(\alpha, \alpha_{m+1}\right)$. Let $Z=\inf \left(n \geq 0: X_{n}=m+1\right)$ be the random variable of the time to the absorbing state $m+1$. The distribution of $Z$ is called a phase-type distribution with representation $(\alpha, T)$. Note that the knowledge of $(\alpha, T)$ is sufficient since

$t^{\prime}=-T 1, \quad \alpha_{m+1}=1-\alpha 1$

The dimension $m$ of $T$ is called the order of the $\mathrm{PH}$ distribution and the transient states $\{1,2,3, \ldots, m\}$ are called the phases. The vector $t^{\prime}$ contains the so-called exit rates.

The cumulative distribution function of the $\mathrm{PH}$ distribution $\mathrm{Z}: \mathrm{PH}(\alpha, T)$ is given by

$$
F_{Z}(z)=1-\alpha T^{z} 1 \quad \text { for } \quad z=0,1,2, \ldots
$$

and its probability density function is

$$
\begin{aligned}
& f_{Z}(0)=\alpha_{m+1} \\
& f_{Z}(z)=\alpha e^{T z} t^{\prime} \quad \text { for } \quad z=1,2,3, \ldots
\end{aligned}
$$

Proof of equation (1), taking transition matrix function $\mathrm{Q}(z)=e^{\mathrm{Q} z}$, we get

$$
\sum_{n=0}^{\infty} \frac{(\mathrm{Q} z)^{n}}{n !}=\sum_{n=0}^{\infty}\left[\begin{array}{cc}
T & t^{\prime} \\
0 & 0
\end{array}\right] \frac{Z^{n}}{n !}=\left[\begin{array}{cc}
e^{T z} & 1-e^{T z} 1 \\
0 & 0
\end{array}\right]
$$

Taking the initial distribution $\alpha, \alpha_{m+1}$ into account and using expression (2) for the transition matrix, we obtain $F_{Z}(z)=1-\alpha e^{T z} 1$

The service time of the systems are following phase-type distribution $\mathrm{PH}(\alpha, T)$ and $T$ is an $m \times m$ matrix. The mean service time is given by $-\alpha T^{-1} 1$. The repair time distribution is following phase-type distribution with representation $(\beta, S)$, where $S 1+S^{\prime}=1, S$ is an $n \times n$ and 1 is the $n$ dimensional column vector with all elements

equal to 1 . The mean repair time is given by $-\beta S^{-1} 1$. Upon the completion of a repair, the system is as new as one.

\section{The Model Description}

In this section, we first describe the system model. Then we derive a quasi-birth and death process of the system. The symbols $\otimes$ and $\oplus$ denote the kronecker product and the kronecker sum of two matrices, respectively. Thus, if $A$ is a matrix of order $m_{1} \times m_{2}$ and if $B$ is a matrix of order $n_{1} \times n_{2}$, then $A \otimes B$ will denote a matrix of order $m_{1} n_{1} \times m_{2} n_{2}$ whose $(i, j)^{\text {th }}$ block matrix is given by $a_{i j} B$. For more details on kronecker products, we refer to Bellman [3]. Before we describe the Markov chain the repairman model, we represent a review of PH-distribution.

The assumption of the system model are as follows

- Working system can fails and goes to repairs with the repair man idle and standby becomes the new working system. The block that represents this transition is $\left(t^{\prime} \alpha \otimes \beta\right)$ of order $(m \times m k)$.

- Working system fails and goes to the repairs queue(repair man busy). The order of the sub-matrix is $m k \times m k$ it is $\left(t^{\prime} \alpha \otimes I\right)$.

- When a repair is completed, the system goes to standby and the repair becomes idle. The working system does not change the phase. The order of the sub-matrix is $(m k \times m)$ and it is $\left(I \otimes S^{\prime}\right)$.

- The repairman becomes busy. The order of the sub-matrix is $(m k \times m)$ and the matrix is $\left(I \otimes S^{\prime} \beta\right)$.

- The transition $0 \rightarrow 0$ is governed but the matrix $T$. The transition $i \rightarrow i(i>0)$ is covered by the matrix $S$, when there are changes among the operational or repair phases.

- The block is $T \oplus S=T_{m} \otimes I_{k}+I_{m} \otimes S_{k}$ order of the sub-matrix is $m k \otimes m k$. The matrix $I$, we denote identity matrices of appropriate orders.

\section{Quasi-Birth and Process}

This matrix represents a Quasi-birth and death process was developed Neuts [7] to solve the stationary state probability for the vector state Markov process with repetitive structure. We develop the steady-state probability. The corresponding transition rate matrix $\Theta$ at this Markov chain has the block tridiogonal form. Consider the generator matrix $Q$ as shown below 


\section{International Journal of Science and Research (IJSR) \\ ISSN (Online): 2319-7064}

Index Copernicus Value (2013): 6.14 | Impact Factor (2014): 5.611

$$
\Theta=\left|\begin{array}{cccccc}
\mathrm{B}_{0} & \mathrm{X}_{0} & & & & \\
\mathrm{~B}_{1} & \mathrm{~A}_{1} & \mathrm{~A}_{0} & & & \\
& \mathrm{~A}_{2} & \mathrm{~A}_{1} & \mathrm{~A}_{0} & & \\
& & \mathrm{~A}_{2} & \mathrm{~A}_{1} & \mathrm{~A}_{0} & \ldots \\
& & & \ddots & \ddots & \ddots
\end{array}\right|
$$

Where $\mathrm{B}_{0}=T, \mathrm{X}_{0}=t^{\prime} \alpha \otimes \beta, \mathrm{B}_{1}=I \otimes S^{\prime}, \mathrm{A}_{0}=t^{\prime} \alpha \otimes I$,

$$
\mathrm{A}_{1}=T \otimes S, \mathrm{~A}_{2}=I \otimes S^{\prime} \beta
$$

Then we have the following stability condition for the QBD process.

\section{Stationary Probability Vector}

We use $\Xi=\left[\Xi_{0}, \Xi_{1}, \Xi_{3}, \ldots\right]$. It is known from Neuts[7], where $\Xi_{j}=(\Xi(j, 1), \Xi(j, 2), \ldots, \Xi(j, k))$ to denote the stationary probability vector, which satisfies the matrical equation $\Xi \Theta=0$, subject to the normalization condition $\Xi 1=1$. For $j=0,1,2, \ldots$ and $\Xi(j, k)$ is the probability of finding the system in the state $(j, k)$ as steady state. This gives the following equation

$$
\begin{gathered}
\Xi_{0} \mathrm{~B}_{0}+\Xi_{1} \mathrm{~B}_{1}=0 \\
\Xi_{0} \mathrm{X}_{0}+\Xi_{1} \mathrm{~A}_{1}+\Xi_{2} \mathrm{~A}_{2}=0 \\
\Xi_{1} \mathrm{~A}_{0}+\Xi_{2} \mathrm{~A}_{1}+\Xi_{3} \mathrm{~A}_{2}=0 \\
\vdots \\
\Xi_{j-1} \mathrm{~A}_{0}+\Xi_{j} \mathrm{~A}_{1}+\Xi_{j+1} \mathrm{~A}_{2}=0
\end{gathered}
$$

Subject to

$$
\sum_{j=0}^{\infty} \Xi_{j} 1=1
$$

In analog with the point situation, it may be shown that there exits a constant matrix $\mathrm{P}$ such that

$$
\Xi_{j}=\Xi_{j-1} \mathrm{P} \quad \text { for } \quad j=2,3, \ldots
$$

The sub-vector $X_{j}$ are said to be geometrically related to each other since

$$
\Xi_{j}=\Xi_{1} \mathrm{P}^{j-1}
$$

If the sub-vectors $\Xi_{0}$ and $\Xi_{1}$ and the rate matrix $\mathrm{P}$ can be found then the remaining sub-vectors of the stationary distribution may be formed using equation (7), substituting from equation (7), we obtain for $j=2,3, \ldots$

$\Xi_{0} \mathrm{P}^{j-2} \mathrm{~A}_{0}+\Xi_{1} \mathrm{P}^{j-1} \mathrm{~A}_{1}+\Xi_{2} \mathrm{P}^{j} A_{2}=0$

ie.,

$\Xi_{1} \mathrm{P}^{j-2}\left(\mathrm{~A}_{0}+\mathrm{P} \mathrm{A}_{1}+\mathrm{P}^{2} \mathrm{~A}_{2}\right)=0$

It is now apparent that $\mathrm{P}$, it is known from Neuts [7] that there exits an $\mathrm{P}$ matrix which is the minimal non-negative solution to the matrix quadratic equation

$\mathrm{A}_{0}+\mathrm{PA}_{1}+\mathrm{P}^{2} \mathrm{~A}_{2}=0$
Given the rate matrix $\mathrm{P}$ and blocks $\mathrm{B}_{0}, \mathrm{X}_{0}, \mathrm{~B}_{1}, \mathrm{~A}_{0}, \mathrm{~A}_{1}$ and $A_{2}$ the system may be solved to obtain $\Xi_{0}$ and $\Xi_{1}$. The computed solution needs to be normalized so that the components of $\Xi$ sum to 1 . In other words, we insist that $\Xi 1=1$. Thus

$\Xi_{0} 1+\sum_{j=0}^{\infty} \Xi_{1} \mathrm{P}^{j} 1=1$

This implies the condition

$$
\Xi_{0} 1+\Xi_{1}\left(\sum_{j=0}^{\infty} \mathrm{P}^{j}\right) 1=1
$$

The eigenvalues of $\mathrm{P}$ lie inside the unit circle, which means that $(I-\mathrm{P})$ is non-singular hence that

$$
\left(\sum_{j=0}^{\infty} \mathrm{P}^{j}\right)=(I-\mathrm{P})^{-1}
$$

This enable us to the normalizing of the vectors $\Xi_{0}$ and $\Xi_{1}$ by computing

$$
\Xi_{0} 1+\Xi_{1} \sum_{j=1}^{N} \mathrm{P}^{j-1} 1=1
$$

and consequently equation (4) and (5) can be expressed as

$\Xi_{0} \mathrm{~B}_{0}+\Xi_{1} \mathrm{X}_{0}=0$

$\Xi_{0} \mathrm{~B}_{1}+\Xi_{1}\left[\mathrm{~A}_{1}+\mathrm{P} \mathrm{A}_{2}\right]=0$

$\Xi_{0} 1+\Xi_{1}[I-\mathrm{P}]^{-1} 1=0$

and dividing the computing sub-vector $X_{0}$ and $X_{1}$ by (9)

$$
\begin{aligned}
& \left(\Xi_{0}, \Xi_{1}\right)\left\lfloor\begin{array}{ccc}
1 & \mathrm{~B}_{0} & \mathrm{X}_{0} \\
(I-\mathrm{P})^{-1} 1 & \mathrm{~B}_{1} & \mathrm{~A}_{1}+\mathrm{P} \mathrm{A}_{2}
\end{array}\right\rfloor \\
& =(1,0)
\end{aligned}
$$

The first column of the included matrix is removed to avoid linear dependency. The removed column is replaced in the normalization condition. Equation (10) is solved for computing $\Xi_{0}$ and $\Xi_{1}$.

\section{Stability Condition}

The drift to higher numbered levels must be strictly less than the drift to lower levels. let $\mathrm{A}=\mathrm{A}_{0}+\mathrm{A}_{1}+\mathrm{A}_{2}$ and $\Xi_{\text {A }} A=0$. The steady state probability vector exits if only if

\section{$\Xi \mathrm{A}_{0} 1<\Xi \mathrm{A}_{2} 1$}

Where $X$ is the invariant probability vector of the matrix $A=A_{0}+A_{1}+A_{2}$. As $A$ is finite, the steadystate probability $\Xi$ of the new CTMC can be easily computed. Then the drift to the next higher level can be described as:

$\sum_{i} \Xi_{i} \sum_{l} \mathrm{~A}_{0}(i, l)=\Xi \mathrm{A}_{0} 1$

and the drift to the next lower level as: 
$\sum_{i} \Xi_{i} \sum_{l} \mathrm{~A}_{2}(i, l)=\Xi \mathrm{A}_{2} 1$

which leads to

$\Xi_{\mathrm{A}}=\Xi$

For this we can show the stability condition for this system to be as following theorem.

\section{Theorem 1 The markov chain in equation(3) is positive recurrent iff \\ $a<b$}

Proof. It is known from Neuts [7] that if the matrix A is of finite the criterion for the matrix in equation(3) to be positive recurrent is the mean drift condition

$\Xi \mathrm{A}_{0} 1<\Xi \mathrm{A}_{2} 1$

The equation that results from multiplying $\left(\Xi_{0}, \Xi_{1}\right)$ by the first column of the corresponding to the normalizing condition. The linear equations given by equation (10) have a unique solution that also satisfies the normalizing condition. If however $A$ is irreducible that vector $\Xi$ exits then this result still holds for the infinite block (see [12]). Therefore we have

$$
\begin{aligned}
& \Xi \mathrm{A}_{0} 1=\left(\xi_{a} \otimes \xi_{b}\right)\left(t^{\prime} \alpha \otimes I\right) 1<\Xi \mathrm{A}_{2} 1 \\
& =\left(\xi_{a} \otimes \xi_{b}\right)\left(I \otimes S^{\prime} \beta\right) 1
\end{aligned} \quad \begin{aligned}
& \Rightarrow\left(\psi_{a} t^{\prime}\right) \otimes\left(\psi_{b} I 1\right)<\left(\psi_{a} I 1\right) \otimes\left(\psi_{b} S^{\prime}\right) \\
& \Rightarrow a(1-b)<(1-a) b=a<b
\end{aligned}
$$

Remark: It can be show that $a=\psi_{a}(t)$ and $b=\psi_{b}(t)$. This is intuitive because $\psi_{a}(1)$ is the stationary probability of being in state 1, which is essential the probability of an working system. The same argument is true for service and repair system. It is known from Neuts [7] that

$$
\begin{array}{lll}
a=\psi_{a} t^{\prime} & \text { and } & 1-a \psi_{a} I 1 \\
\text { similarly } & & \\
b=\psi_{b} s^{\prime} & \text { and } & 1-b \psi_{b} I 1
\end{array}
$$

\section{Queue Length and Waiting Time distribution of Repair}

A very important performance measure of interest is the distribution of the number of working system of arbitrary time, which is obtained from the stationary vector $\Xi$, where $\Xi \Theta=0$ and $\Xi 1=1$. In section 4 and Neuts [6], we can obtain the vector $\Xi$ and all information about the queue length. For example, the mean number of working $\operatorname{system}\left(\mu_{L}\right)$ in the repair facility and its mathematical expression is

$\mu_{L}=\sum_{j=1}^{\infty}(j-1) \Xi_{j} 1=\Xi_{1}[I-\mathrm{P}]^{-2} 1$

The mean number of system in queue for repair is

$$
\mu_{q}=\sum_{J=1}^{\infty} \Xi_{J} 1=\mu_{L}-\left(1-\Xi_{0} 1\right)
$$

\section{Availability}

We conclude the availability and the rate of occurrence of failures, the mean number of non-working system, and explicit algebraic formulae are given. All the measures will calculated in the stationary regime. The availability is the first measure to be calculated in the repairable system. It is well known that the availability is the probability that the system will be service. Analytically, the expression of the availability is exactly the normalization condition, that is,

$$
A=\sum_{i=0}^{\infty} \Xi_{i} 1=\Xi_{0} 1+[I-\mathrm{P}]^{-1} 1=1
$$

Consequently, The non-availability is given by $\bar{A}=1-A=0$, which is the probability that the system is working.

\section{Rate of Occurrence of failure}

A failure can occurs when the system occupies some of the states $i=0,1,2, \ldots$, when the system is in state 0 , the failure occurs by absorption in the distribution $\operatorname{PH}(\alpha, T)$ and repairman is idle. The space of repair must not change. Thus, the rate of occurrence of failures is given by the expression

$b=\Xi_{0} t^{\prime}+\sum_{i=1}^{\infty} \Xi_{i}\left(t^{\prime} \otimes 1\right)=\Xi_{0} t^{\prime}+\Xi_{1}[I-\mathrm{P}]^{-1}\left(t^{\prime} \otimes 1\right)$

Multiplying on the right the first equation in (3) determines a relation between $\Xi_{0}$ and $\Xi_{1}$, and the rate of occurrence of failure can be expressed in terms of $X_{1}$ as follows $b=\Xi_{1}\left[1 \otimes S^{\prime}+(I-\mathrm{P})^{-1}\left(t^{\prime} \otimes 1\right)\right](10)$

\section{Numerical Examples}

In this section we apply the calculation performed above the practical case. The methods were implemented in matlab programme. We consider the following represents for the $\mathrm{PH}$-distribution of the service time and repair time $\alpha(1,0)$ and $\beta(1,0,0)$

$T=\left(\begin{array}{cc}0.3 & 0 \\ 0 & 0.4\end{array}\right) ; S=\left(\begin{array}{ccc}1 & 1 & 0 \\ 0 & 1.5 & 1.5 \\ 0 & 0 & 2\end{array}\right)$

The matrix $T$ indicates the working system has an operational time that undergoes successive decorating phases. The order of $T$ and $S$ are, respectively $m=2$ and $n=3$. Consequently, the matrices $\mathrm{A}_{0}, \mathrm{~A}_{1}$ and $\mathrm{A}_{2}$ are of order $6 \times 6 ; \mathrm{B}_{01}$ and $\mathrm{B}_{10}$ are of order $2 \times 6$ and $6 \times 2$. The availability and the rate of occurrence of different 


\section{International Journal of Science and Research (IJSR) \\ ISSN (Online): 2319-7064}

Index Copernicus Value (2013): 6.14 | Impact Factor (2014): 5.611

failures, for the system with different number of working system. The availability decreases slightly when the number of working system increases and stabilizes of around the $91.7 \%$. The different rate of occurrence of failures change slowly with ' $n$ '. The sub-vector of the stationary probability vector are given below, where the value $\leq 10^{-4}$ are substitute by a asterisk,

$$
\begin{aligned}
& \Xi_{0}=(0.3500,0.2610) \\
& \Xi_{1}=(0.1409,0.0805,0.0023,0.0013,0.0004,0.0002) \\
& \Xi_{1}=(0.0011,0.0004,0.0003,0.0000,0.0000,0.0000)
\end{aligned}
$$

The performance measures calculated in this model depend on the sub-vectors $\left(\Xi_{0}, \Xi_{1}\right)$, they can be explicitly determined, and they are $b=0.001$

The number of queued and waiting for repair is $\mu_{q}=0.0776$

The mean total expected in the repair facility is $\mu_{L}=0.3651$

The small value are expected for the number of system in repair, since the mean time of the working system is substantially longer than the expected time to complete a repair.

\section{Conclusion}

In this paper, we have a comparative analysis for a repair problem where both the systems and the repairman can fail. The repair times of the systems and the service times of the repairman are modelled using different $\mathrm{PH}$-distribution. We include recent and faster computational methods for practical applications of QBD processes. Calculate the stationary probability vector and to determine an appropriate from for the iterative numerical calculate of the blocks of the matrix $\mathrm{P}$ in some particular simple cases, as in the model Erlang/ph/1. Our results can be treated as performance evaluation tool for the concerned system which may be suited to many congestion situations arising in many practical applications encountered in computer and communication systems, distribution and service sectors, production and manufacturing system, etc.,

\section{References}

[1] Chakravarthy .S, Reliability Analysis of a Parallel System with exponential Life Time and Phase-Type repairs, Transp. Sci., OR Spekrum, 5; pp25-32(1983).

[2] Chakravarthy .S and Natarajan .R, A Study of n-unit System Operational in a Random Environment, Eur J.Oper.Res, 66; pp223-236(1999).

[3] Bellman .R, Introduction to matrix analysis, McGraw Hill, New York(1960).

[4] Latouche.G and Ramaswami.V, Algorithmic Reduction for Quasi-Birth and Death Process, J Appl.Prob, 30;pp650-674(1993).

[5] Latouche .G and Ramaswami .V, Introduction to matrix methods in Stochastic Modelling, Philadelphin: ASA-
SIAM Series on Statistics and Applied Probability (1999).

[6] Neuts.M.F, Probability Distributions of phase-type, In: Liber Amicorum Professor Emiritus H.Florin. Department of Mathematics, University of Lounvain, Belgium, pp183-206(1978).

[7] Neuts .M.F, Matrix Gometric Solution in Stochastic Models: An Algorithmic Approach, John Hopkins Univrsity Press, Baltimore(1981).

[8] Neuts .M.F and Meiee .K.S, On the Use of Phase-Type Distribution in Reliability Modelling with two Components. OR Spekrum, 2; pp227-234(1986).

[9] Neuts .M.F, Perez-Ocon .R and Terres Castro .I, Repairable Models with Operating and Repair Times Governed by Phase-Type Distribution. Advances in Applied Probability, 32; pp468-479(2000).

[10] Ramaswami .V and Taylor .A.G, Some Properties of the Rate Operators in Level Dependent Quasi-Birth and Death Processes with a Countable Number of Phases, Stochastic Models,12(1); pp143-164(1996).

[11] Sivazlian .B.D and Wang .K.H, Diffusion approximation to the $G / G / R$ machine repair problem with warm standby spares, Nav Res Logistics, 37; pp753772(1990).

[12] Tweedie .R.L, Operator-Geometric Stationary Distribution Markov Chaine with Applications to Queueing Models, Adv.in Appl.Probab,42(2); pp368392(1982).

[13] Wang .K, Profit analysis of the machine repair problem with cold standbys and two modes of failure, Microelectron Reliab,34; pp1635-1642(1994).

[14] Wang .K and Lee .H, Cost analysis of the coldstandby $M / M / R$ machine repair problem with multiple modes of failure, Microelectron Reliab,38; pp435441(1998).

[15] Wartenhorst .P, N Parallel queueing systems with server breakdowns and repair, Eur J Oper Res, 82; pp302322(1995).

[16] van der Duyn .S.F.A and Wartenhorst .P, Two machine repair model with variable repair rate, Nav Res Logistics, 40; pp495-523(1993). 